\title{
Cloning, expression, purification and functional analysis of a specific multi-epitope protein from multi drug resistance Acinetobacter baumannii
}

\author{
Zahra Davoudi ${ }^{1}$, Amirhossein Taromchi ${ }^{2}$, Bahram Kazemi ${ }^{3}$, Mojgan Bandehpour ${ }^{1,3^{*}}$, Nariman Mosaffa ${ }^{4}$ \\ ${ }^{I}$ Department of Medical Biotechnology, School of Advanced Technologies in Medicine, Shahid Beheshti \\ University of Medical Sciences, Tehran, Iran \\ ${ }^{2}$ Department of Medical Biotechnology and Nanotechnology, School of Medicine, Zanjan University of \\ Medical Sciences, Zanjan, Iran \\ ${ }^{3}$ Cellular and Molecular Biology Research Center, Shahid Beheshti University of Medical Sciences, Tehran, \\ Iran \\ ${ }^{4}$ Department of Immunology, School of Medicine, Shahid Beheshti University of Medical Sciences, Tehran, \\ Iran
}

Received: April 2021, Accepted: September 2021

\begin{abstract}
Background and Objectives: Immunization is a promising strategy to combat against the life-threatening infections by Multi Drug Resistance Acinetobacter baumannii. In this study, we directed to design and evaluate the efficacy of a recombinant multi-epitope protein against this pathogen.

Materials and Methods: Epitopes prediction was performed for candidate proteins OmpA and BAM complex (BamA, BamB, BamC, BamD, BamE) from A. baumannii, using immune-informatics tools with high affinity for the human HLA alleles. After expression and purification of the recombinant protein, its functional activity was confirmed by interaction with positive sera.

Results: Cloning and expression of the desired multi-epitopes protein were verified. Circular Dichroism study showed the secondary structure and proper refolding of the recombinant protein was achieved and matched with computational prediction. There was a significant interaction between designed protein with antibodies presented in ICU patients' and staff's sera. Conclusion: The interaction of the recombinant protein with patients' sera antibodies suggests that it may be a promising determinant protein for immunization against of MDR A. baumannii.
\end{abstract}

Keywords: Multi drug resistant; Acinetobacter baumannii; Recombinant multi-epitope protein (rMEP); OmpA; BAM complex

\section{INTRODUCTION}

Acinetobacter baumannii is an opportunistic nosocomial Gram-negative bacilli that causes a various ranges of infections in hospitalized patients in
ICU (intensive care units) and injured war soldiers (1). Currently, antibiotics are the only treatment for infections caused by this pathogen. Resistant $A$. baumannii strains to all available antibiotics even Carbapenems, has been reported worldwide (2).

"Corresponding author: Mojgan Bandehpour, Ph.D, Department of Medical Biotechnology, School of Advanced Technologies in Medicine, Shahid Beheshti University of Medical Sciences, Tehran, Iran; Cellular and Molecular Biology Research Center, Shahid Beheshti University of Medical Sciences, Tehran, Iran. Tel: +98-21-22439957Ｆax: +98-21-22439956 Email: m.Bandehpour@sbmu.ac.ir 
However, colistin is the last treatment resort for MDR A. baumannii but its use has been limited because of drug side effects such as neurotoxicity and nephrotoxicity. Since infections by multi- or pandrug resistant $A$. baumannii has created high mortality rates, prolonged hospitalization and financial cost of treatment $(1,3,4)$, achieving an immunogenic protein can play an important role in vaccine development to prevent multi drug resistant infections (5). According to the previous studies, vaccination with whole cell pathogen, outer membrane complex Ata (6, 7), FilF (8), capsule components (9), Bap (10) and outer membrane vesicles (11) have been created immune responses against $A$. baumannii. Ideally, an appropriate vaccine candidate antigen is conserved in clinical strains that play an important role in pathogenesis of microorganism. Outer membrane proteins (Omps) act as efficient protective antigens because they are capable of stimulating host humoral immune system and providing prevention against bacterial challenges (12-14). It has an important role in adherence and interaction with eukaryotic cells, biofilm formation $(15,16)$, host cell apoptosis (17), stimulation of immune responses, and biogenesis of outer membrane vesicles (16). In Gram negative bacteria $\beta$-Barrel Assembly Machine (BAM) is a protein complex (18) that assemble folding and insertion of $\beta$-barrel proteins into the bacterial outer membrane. Because of crucial role of complex BAM in outer membrane protein assembling, it is highly conserved among the Gram negative bacteria. The BAM protein is composed of BamA, BamB, BamC, BamD and BamE proteins $(19,20)$. Due to the lack of a licensed vaccine against $A$. baumannii, different proteins and peptides might be tested to achieve an appropriate and effective vaccine candidate. According to the recent studies regarding the A. baumannii, new combination antigens OmpK/Omp22 (21), FilF/ NucAb (22), OMV/Bap, and OmpA/Bap (23) have produced the partial to complete protection against

bacteria in mice.

The sequencing of microbial genomes has been provided the potential antigens of each pathogen (24). Recently, a new method based on immuneinformatics for prediction of antigenic epitopes from numerous proteins is established. The advantages of the epitope-based proteins are high specific, no pathogenicity due to failure to return to the active state in the host, being financially and temporally cost-effective, reducing adverse effects in comparison with killed and attenuated form of vaccines (25). Therefore, we designed our study based on the novel recombinant multi-epitopes protein called rMEP consists of the immunogenic epitopes fragments from OmpA, BamA, BamB, BamC, BamD and BamE determinant antigens of A. baumannii. Subsequently, reaction of the recombinant multi-epitopes protein with sera from ICU staff and recovering patients with $A$. baumannii infections was evaluated by ELISA, and Western blotting.

\section{MATERIALS AND METHODS}

Study design and ethics statement. The current research was carried out in two parts. In the first part, we designed and evaluated the cloning, expression and purification of the recombinant multi-epitope protein, also we have analyzed the secondary structures and appropriate folding of the purified rMEP protein. In the second part, we confirmed reaction of the rMEP protein with sera of ICU staff and recovering patients with $A$. baumannii using ELISA and Western blotting test.

Required permissions and consent was taken from university hospitals management as well ICU staff and patients (Ethics Committee number IR.SBMU. RETECH.REC.1399.195).

Bacterial strains and plasmid vector. MDR $A$. baumannii clinical isolate strain, was prepared from the microbial collection of Microbiology Department, Shahid Beheshti University of Medical Sciences. The prepared MDR A. baumannii was found to be non-susceptible to antibiotics includes cefotaxime, ceftazidime, meropenem, imipenem, jentamicin, amikacin, ciprofloxacin, cefepime, piperacillin, piperacillin/tazobactam, co-trimoxazole, tetracycline and only it is susceptible to colistin. In addition, the strain harboured $b l a_{\text {OXA-51- }}, b l a_{\text {OXA-23- }}$, and $b l a_{\text {OXA- }}$ 24-like genes (26). E. coli strain Top10 was used for the cloning of construct and E. coli strain BL21 (DE3) (Stratagene, La Jolla, CA) as well pET26b vector were used for the expression of the recombinant protein.

Construction of the recombinant protein: In silico design and evaluation of the rMEP protein construct. The full-length proteins sequences of OmpA (WP_000777878.1), BamA (WP_001983709.1), BamB (WP_001072121.1), BamC (WP_001297320.1), 
BamD (WP_000056813.1) and BamE (WP_001170994.1) antigens of A. baumannii, the highly MDR AYE strain were retrieved from UniProt (https://www.uniprot.org/proteomes/UP000002446) in FASTA format. Multiple sequences alignment performed between the sequences from different strains using Clustal W2 software (https://www.ebi.ac.uk/Tools/msa/clustalo/) to identify conserved fragments in A. baumannii sub species reported sequences from different areas. We applied online servers to predict B-cell and T-Cell - MHC I and II binding epitopes. Prediction of B-cell epitopes performed using IEDB (http://tools.iedb.org), ABCpred (http://crdd.osdd.net/raghava/abcpred/). To identify T-cell epitopes several servers such as IEDB, ProPred (http://crdd.osdd.net/raghava/propred1/) with parameters for human HLA allele class II (HLADRB1*11, 13, 15, 03, 04) employed. The screened B-cell and T-cell epitopes were joined using GGGGS linkers to design protein construct that named rMEP.

Computed physical and chemical parameters of the rMEP. Physical and chemical characters including molecular weight, theoretical isoelectric point (pI), extinction coefficient, half-life, instability index, grand average of hydropathy (GRAVY) and total number of positive and negative residues were computed using the ProtParam tool (http://expasy.org/tools/protparam.html) . Antigenicity, solubility and flexibility of the rMEP was considered in epitopes prediction by IEDB server.

Prediction, and validation of the rMEP protein structure properties. Prediction of secondary, tertiary structure and modeling of rMEP were achieved using I-TASSER (http://zhanglab.ccmb.med.umich.edu/I-TASSER) online web server.

The geometry quality of designed protein was validated using Ramachandran analysis at (https://servicesn.mbi.ucla.edu/PROCHECK/) and Z-score of ProSA server at (https://prosa.services.came.sbg.ac.at/prosa.php) and confirmed via Ram- achandran and Z plots.

Codon usage and mRNA structure optimization. The final amino acid sequence was reverse translated into nucleotides and codons were subsequently considered based on E. coli BL21 codon usage by JCAT server (http://www.JCAT.com/). At the end, 3D folding of mRNA, was predicted by mfold web server (http://unafold.rna.albany.edu/q=mfold). Efficiency of translation was analyzed based on structural stability and Gibbs free energy $(\Delta \mathrm{G})$. Finally, rMEP gene was chemically synthesized by Generay CO. (Shanghai, China).

Gene cloning, expression, and purification of the rMEP. The amplification of the synthetic gene was performed using primers (forward primer $5^{\prime}$ ggatccatgctgggctatacctttcagg $3^{\prime}$ and revers primer $5^{\prime}$ aagcttcgcgctcacgcggccgttatc $3^{\prime}$ ). The BamHI and HindIII restriction sites were added to the 5' and 3 ' ends of the sequence using PCR. The PCR product was cloned into $\mathrm{pET} 26 \mathrm{~b}$ expression vector after double digestion. The vector transformed chemically into competent E. coli BL21 (DE3). Positive colonies were confirmed by restriction enzyme digestion and PCR. The transformed bacteria were grown in LB broth and screening was carried out by resistance to kanamycin antibiotic $(30 \mu \mathrm{g} / \mathrm{ml})$. For induction of the protein expression we must use $1 \mathrm{mM}$ isopropyl $\beta$-D-1-thiogalactopyranoside (IPTG) (Thermo Scientific, USA) after reaching OD at $600 \mathrm{~nm}$ to 0.4- 0.6. Then, the cells were harvested by centrifugation and treated with lysis buffer (100 mM NaH2PO4, 10 mM Tris-HCL, 8M urea, $\mathrm{pH}$ 8.0) and followed by sonication on ice in the presence of PMSF (1 mM) as a protease inhibitor. The Bacterial lysate was centrifuged and the supernatant was purified by a Ni-NTA affinity column (Qiagen, Hilden, Germany) based on the standard protocol. The purified protein was refolded to the active form by stepwise urea removal using Amicon-30KDa filter (washing the purified protein with buffers without urea) and concentration of protein was estimated by Bradford assay. Finally, the purified protein was visualized by $12 \%$ SDS-PAGE (Coomassie brilliant blue staining) and identified by Western blotting. In order to Western blot analysis, after SDS-PAGE, the separated protein was transferred on nitrocellulose membrane using a semidry transfer system (Bio-Rad, USA). After blocking of the membrane with 3\% skim milk in PBST (PBS 1\% + Tween20), it was washed with PBST. The blocked membrane was incubated with anti-His tag antibody alkaline phosphatase (ALP) conjugated (Sigma-Aldrich, USA) then membrane was washed in PBST and developed with 3, 3' diaminobenzidine tetrahydrochloride (DAB) (Sigma-Aldrich, USA). 
Circular dichroism spectrophotometry. To identify the secondary structures and proper folding of the purified protein we used far-UV circular dichroism (CD) spectroscopy, JASCO J-810 Spector (Japan, Tokyo). The samples were prepared with a concentration of $0.5 \mathrm{mg} / \mathrm{ml}$, in PBS buffer. The samples and controls in the quartz cuvette with path length $1 \mathrm{~mm}$ were scanned in the far-UV region 180- 240 and speed of $200 \mathrm{~nm} / \mathrm{min}$ at room temperature. Data quantification was carried out by the CAPITO web server (https://data.nmr.uni-jena.de/capito/index.php). The results were compared with the in silico prediction data.

Serum sampling. 20 blood samples were obtained from university hospitals ICU staff, 20 samples from patients with a nosocomial infection as well as 20 samples from healthy people were prepared.

Human sera were analyzed using an optimized indirect ELISA for the presence of specific total antibodies against $A$. baumannii pathogen. The cut-off level was obtained using the mean optical density (OD) of triplicate wells corresponding to the last serum dilution of the healthy group. Sensitivity and specificity of the cut-off was determined.

Confirmation of the collected sera by A. baumannii ELISA. The total antibody level of all serum samples against $A$. baumannii lysate was measured using indirect Enzyme-Linked Immunosorbent Assay (ELISA). The 96-well micro plate (SPL Life Sciences, Korea) was coated by $0.5 \mu \mathrm{g}$ of the pooled clinical isolated A. baumannii and diluted sera (1:50 to 1:6400) were identified by the ELISA assay according to our previous study method (27). E. coli and PBS used as negative controls. The cut-off value was determined by 20 negative serum samples ELISA results. The patients' sera were analyzed based on the cut-off value. They were used for additional assays.

Recombinant multi-epitope protein in interaction with antibodies: Pre-absorption of the serum samples. To avoid background reactivity and improve ELISA specificity, a pre-absorption step was performed on serum samples. For this purpose, sera were incubated with $E$. coli (Top10) lysate at $37^{\circ} \mathrm{C}$, 2 hours, and overnight at $4^{\circ} \mathrm{C}$. The sera were centrifuged $10 \mathrm{~min}, 1000 \mathrm{rpm}$ at $37^{\circ} \mathrm{C}$ and the supernatant was collected and dilute with galactose $0.2 \mathrm{M}$ for using in ELISA assay (28).
Reaction of the rMPE with human serum antibodies. Reaction of positive sera to recombinant multi- epitope protein was determined by ELISA. Briefly, the purified protein $(0.5 \mu \mathrm{g} / \mathrm{ml})$ was coated in 96-well micro plate and incubated overnight at $4^{\circ} \mathrm{C}$. After washing and blocking steps, the plate incubated with serial dilutions (1:10 to 1:6400) of each serum sample from ICU staff, patients and control groups. Then the plate washed and incubated with anti- human HRP conjugated antibody (Abcam, UK). After washing, TMB (Tetra Methyl Benzidine) was used as substrate and reaction was stopped by $2 \mathrm{~N} \mathrm{H} 2 \mathrm{SO} 4$. The absorbance of each well was measured at $450 \mathrm{~nm}$ using an ELISA reader. E. coli and PBS used as controls.

Western blot analysis. Western blot analysis was performed using rMEP protein. After SDS-PAGE, the separated protein was transferred on to nitrocellulose membrane using a semidry transfer system (Bio-Rad, USA). After blocking of the membrane with 3\% skim milk in PBST (PBS 1\% + Tween20), it was washed with PBST. The blocked membrane was incubated with the pool of positive sera in equal proportions, as a first antibody, at a dilution of 1:1000. Anti-human IgG that conjugated with horseradish peroxidase (HRP) (Sigma-Aldrich, USA) was used as a secondary antibody that developed with NBT-BCIP (Sigma-Aldrich, USA).

Statistical analysis. Statistical analyses were performed using GraphPad Prism version 5.00 for Windows, GraphPad Software, La Jolla California USA, www.graphpad.com and Origin(Pro), Version Number 2019b. Origin Lab Corporation, Northampton, MA, USA. Data was analyzed by student t-test, Oneway analysis of variance (ANOVA), Tukey HSD post hoc and bivalent Pearson correlation tests. Results were reported as mean \pm standard deviation (SD). P value $<0.05$ were considered statistically significant.

\section{RESULTS}

Design and analysis of the multi-epitopes protein. The sequences of selected OmpA, BamA, BamB, BamC, BamD and BamE proteins of A. baumannii were retrieved from the Uniprot. Based on the results of multiple sequence alignments between several genes belong to A. baumannii different strains in 
data bank, we selected the homologous sequences for epitope mapping. Based on the results of the B-cell epitopes prediction, a total of seven linear epitopes of the proteins were selected with fixed length 20 aa and best score were selected (Table 1). The T-cell epitopes prediction was considered with fixed length 15 aa and $100 \%$ conservancy level among the A. baumannii strains. Seven low- percentile -rank (percentile rank <1.5) epitopes were selected for good binding peptides (Table 1). Linker consisting GGGGS was introduced between epitopes for efficient and flexible separation.

The designed amino acid sequence (rMEP) was presented as followed:

MLGYTFQDSQHKVAEKLSEYPGGGGSMLVAAPLAAADASRLGGGGSALVSAMAAVQQAYAADDFVVGGGGSNAVKQLLLRKYGNAGGGGGSDANGDSQYDTEKGECKVPEEGGGGSKSGIKYQLSALPLGGGGGGSPVVLGSDLIVGDLDGVLHLIGGGGSVAAYRGKQRLWEKKVGGGGSYKITMLALSLGVASAFVGCSGGGGSKGEVAAYRGKQRLWEGGGGSSGKEETEETTNTPVEAPKSEGGGGSQNFVDLIRRFPSSQYGGGGSKLVLTLFVTSLLAGCSIFGVGGGGSFQQVRFLLGSPTVTDGGGGSSSSEAPSASPADNEADDAAQGGGGSKSATDAANAAQNRGGGGSYKITMLALSLGVASAFVGCSGGGSYQNFVDLIRRFPSSQGGGGSTTAFGAAVVGGDNGRVSA

Underlined fragments were linker sequences.

Physicochemical properties and antigenicity of the rMEP. ProtParam results showed that the de- signed protein contained 421 amino acids and the average molecular weight and theoretical $\mathrm{pI}$ of it were $44.5 \mathrm{kDa}$ and 6.08 respectively. In addition, ProtParam results estimated half-life in vitro and in vivo (Escherichia coli) 30 and 10 hours, respectively. The instability index (II) was computed to be 37.25 . This classifies the multi-epitope as stable protein. The aliphatic and GRAVY indexes of poly epitope were calculated 83.11 and -0.178 , respectively. In addition, rMEP should had appropriate properties in term of antigenicity, allergenicity, solubility, and toxicity. The overall antigenicity of the rMEP was esteemed 0.52 .

Computational properties of the secondary and tertiary structures of the rMEP. The results of secondary structures prediction indicated that multi-epitope consisted of $41.93 \%$ alpha helix, $45.06 \%$ random coil and $13.01 \%$ extended strand without any beta turn in secondary structures (Fig. 1). Tertiary structure prediction of protein was performed by I-TASSER server (Fig. 1). Confidence score (C-score), template modeling score (TM-score) and root-mean-square deviation (RMSD) for 3D models were -2.66, 0.41 \pm 0.14 , and $13.3 \pm 4.1 \AA$, respectively. The stereo chemical quality of above I-TASSER models were analyzed by Ramachandran plot (29). The results revealed that $54.9 \%, 32.5 \%$, and $12.6 \%$ of residues in multi-epitope protein were located in the favorite, allowed and outlier regions, respectively. Also the Z-score of this model obtained from ProSA web was -1.5 that was in the range of native conformations (Fig. 1).

Table 1. Predicted and selected linear B-cell and T-cell - MHC I and II Binding epitopes of OmpA, Bam A, Bam B, Bam C, Bam D, Bam E of A. baumannii via IEDB server.

\begin{tabular}{llccc}
\hline Protein & B-cell Epitope & IEDB score* & T-cell Epitope & IEDB score** \\
\hline OmpA & LGYTFQDSQHKVAEKLSEYP & 1.133 & MLVAAPLAAADASRL & 0.54 \\
BamA & ALVSAMAAVQQAYAADDFVV & 1.104 & NAVKQLLLRKYGNAG & 0.19 \\
& DANGDSQYDTEKGECKVPEE & 1.035 & KSGIKYQLSALPLGG & 0.7 \\
\multirow{2}{*}{ BamB } & PVVLGSDLIVGDLDGVLHLI & 1.124 & VAAYRGKQRLWEKKV & 1.14 \\
& TTAFGAAVVGGDNGRVSA & 1.520 & & 1.48 \\
BamC & YKITMLALSLGVASAFVGCS & 1.099 & KGEVAAYRGKQRLWE & 1.21 \\
& SSSEAPSASPADNEADDAAQ & 1.302 & KSATDAANAAQNR & 0.13 \\
BamD & SGKEETEETTNTPVEAPKSE & 1.092 & QNFVDLIRRFPSSQY & 0.78 \\
& YKITMLALSLGVASAFVGCS & 1.230 & YQNFVDLIRRFPSSQ & 1.48 \\
\hline
\end{tabular}

* IEDB B-cell epitope scores show antigenicity, accessibility, flexibility, hydrophilicity of prediction epitopes. High score is better. ** IEDB T-cell epitope scores show IC50 values for peptides binding to specific MHC molecules. Low score is reliable. 
A

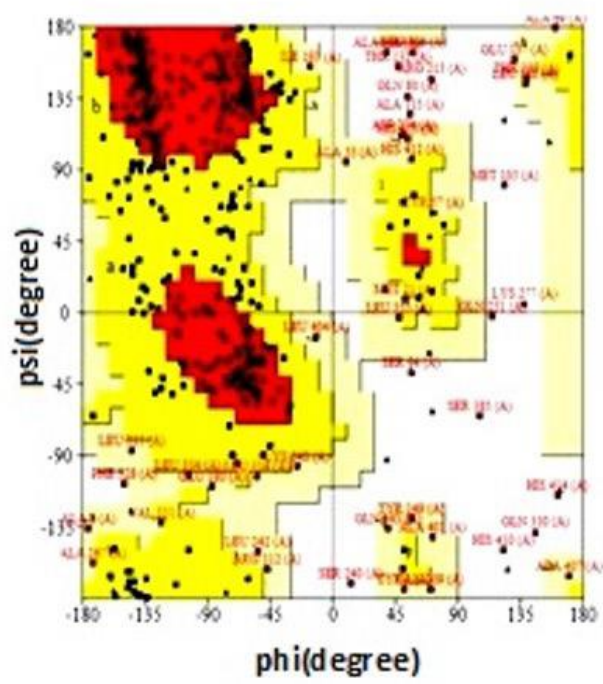

B

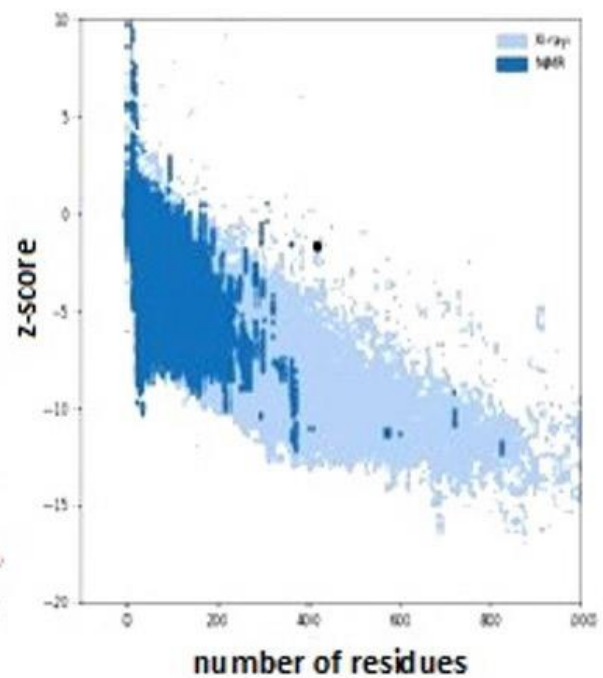

C

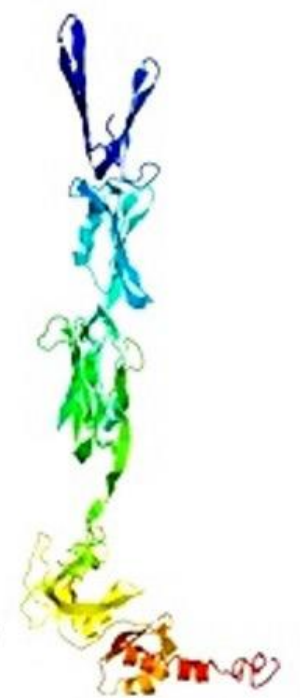

Fig. 1. rMEP 3D structure analysis. A) Ramachandran for validation geometry quality of multi-epitope protein structure. B) The $\mathrm{Z}$ plot of this model was -1.5 that was in the range of native conformations. C) Prediction of the 3D structure of multi-epitope protein using I-TASSER server with C-score 2.66, Estimated TM-score $0.41 \pm 0.14$ and Estimated RMSD $13.3 \pm 4.1 \AA$

Validation of the mRNA structure. After reverse translation of the rMEP, prediction of mRNA structure was carried out by mfold server. The results of mfold showed that $\Delta \mathrm{G}$ of the best predicted structure was $-353.5 \mathrm{kcal} / \mathrm{mol}$ and the $5^{\prime}$ end of the sequence has not any stable hairpin or pseudoknot. Finally, the multi epitope DNA sequence with LC521880 accession number was released in the DDBJ data bank.

Cloning, expression and confirmationof the rMEP. The synthetic designed gene was cloned successfully into pET26b as an expression vector. The existence of fragment in recombinant vector, were confirmed by restriction analysis (Fig. 2). Recombinant protein was successfully expressed in E. coli BL21 (DE3). Optimum expression was obtained with $1 \mathrm{mM}$ IPTG and incubation time of 5 hours at $37^{\circ} \mathrm{C}$. Recombinant protein was mostly expressed as inclusion body and purified under denaturing condition by Ni-NTA affinity chromatography. The purified protein was analyzed by SDS-PAGE and Western blotting (Fig. 3A). The result showed a specific band with a molecular weight of approximately 44.5 KDa. Recombinant expression of the rMEP was performed by Western blot analysis using anti His tag antibody (Fig. 3B).

Circular dichroism spectrophotometry. To monitor conformational changes in purified rMEP sec-

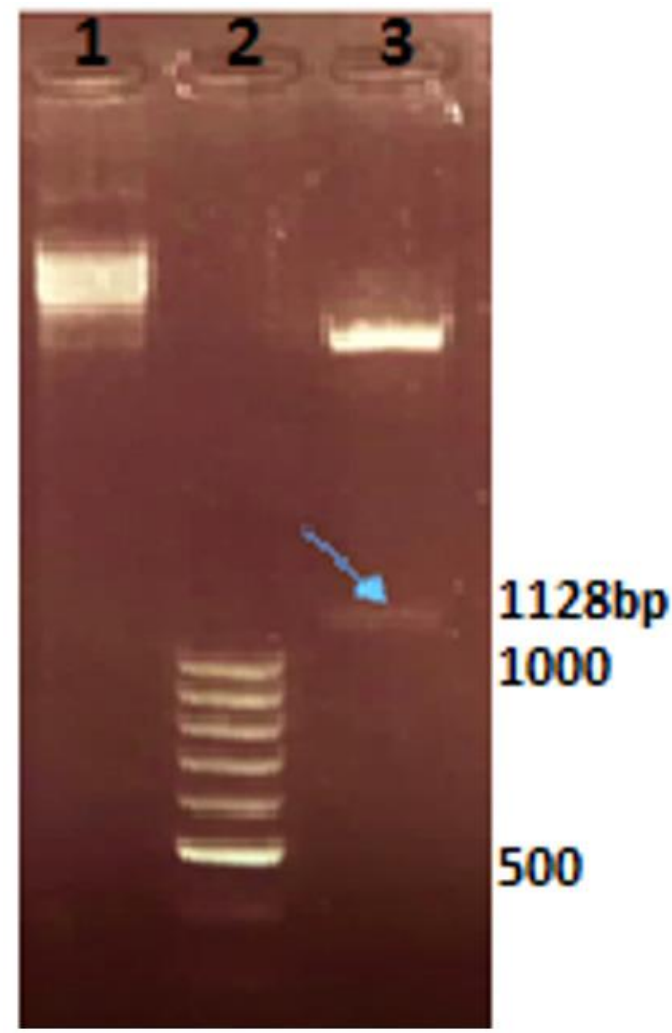

Fig. 2. Double digestion of the recombinant plasmid using BamHI and HandIII restriction enzymes. lane 1, Undigested pET26b-rMEP; lane 2, 100bp DNA Ladder; lane 3, Double digestion of the pET26b- rMEP sequence, insertion size is $\sim 1128 \mathrm{bp}$. 
ondary structures, far-UV CD analysis was performed. The molar ellipticity [ $\theta]\left(\mathrm{deg} \mathrm{cm}^{2} \mathrm{~mol} \mathrm{-1}\right)$ at $208 \mathrm{~nm}, 222 \mathrm{~nm}$ and at the ratio of 208/222 showed $\alpha$-helix content. Slight changes were observed in the secondary structures of the protein compared to in silico analysis (Table 2). The data of the CD spectra of recombinant protein in the far UV range was shown in Table 2 and confirmed the rMEP secondary structures were in agreement with its in silico prediction. It seems that part of the random coil predicted structure of the rMEP protein has been converted to beta-strand structure in vitro due to the presence of ions in the buffer or other technical changes.

Confirmation of the collected positive sera. $\mathrm{Hu}-$ man positive sera were analyzed using an indirect ELISA with extracted $A$. baumannii lysate. The cutoff level was obtained $(\mathrm{OD}=0.22)$ using the mean optical density (OD) of triplicate wells corresponding to the last serum dilution of the healthy group. Sensitivity and specificity of the cut-off was determined $65 \%$ and $90 \%$ respectively. The results revealed that 4 of the 20 patient's sera were negative and that the remaining samples were positive. ICU staff's sera assessment has shown that 18 of the 20 samples were positive and 2 samples were negative. All healthy group' sera analysis did not show any reaction in ELISA. The data reported the mean of OD450 \pm SD with $\mathrm{P}>0.001$ (Fig. 3).

Evaluation of rMEP in interaction with positive sera antibodies: Western blot analysis. Western blot analysis of purified rMEP using pooled positive sera as primary antibody were developed with secondary anti-human IgG HRP-conjugated that visualized with NBT-BCIP (Nitroblue tetrazolium and 5-Bromo-4-chloro-3-indolyl phosphate). The results showed favorite band with desired molecular weight $\sim 45.5 \mathrm{KDa}$ (Fig. 3C).

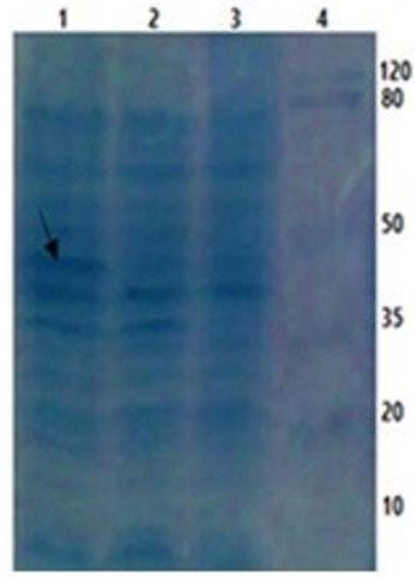

A

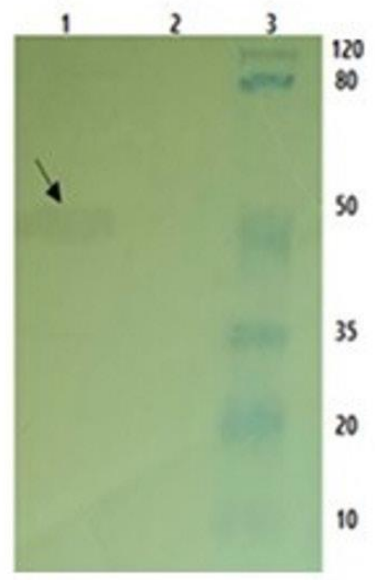

B

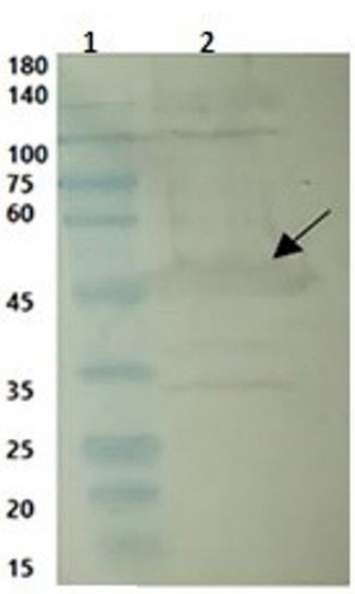

C

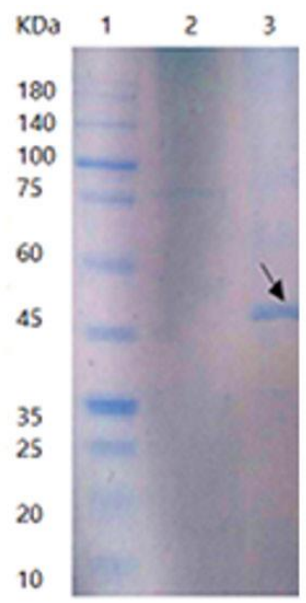

D

Fig. 3. SDS-PAGE and Western blot analysis of multi-epitope protein. (A) Expression analysis of rMEP. lane 1: induced BL21-rMEP 45.5KDa; lane 2 un induced cells lysates, lane 3 control; lane 4: Standard protein marker. (B) Western blot analysis of rMEP using anti His tag antibody. lane1: rMEP with MW 5.5KDane 2: un induced E. coli BL21 lysate. Lane 3: Standard protein marker. (C)Interaction of the rMEP and positive sera by Western blot analysis. Lane 1. Standard protein marker; lane 2. induced cells lysates. (D) Purification analysis of protein. lane 1: Standard protein marker; lane 2: Washed buffer; lane 3: Eluted protein $\sim 45.5 \mathrm{KDa}$

Table 2. The compared secondary structure of the purified protein predicted using GOR4 server with the secondary structure that revealed by Circular Dichroism

\begin{tabular}{lccc}
\hline Secondary structure & Alpha-helix $(\boldsymbol{\%})$ & Beta-strand $(\boldsymbol{\%})$ & Random coil $(\boldsymbol{\%})$ \\
\hline GOR4 predicted & 41.93 & 13.01 & 45.06 \\
Circular Dichroism & 40.8 & 21.1 & 38.1 \\
\hline
\end{tabular}


ELISA. Specific interaction between rMEP and total serum antibodies was measured by indirect ELI$\mathrm{SA}$ in different dilutions of serum obtained from ICU patients and staff (Fig. 4A). A significant increase was observed in the reaction between serum dilution $1 / 100$ and $0.5 \mu \mathrm{g}$ rMEP multi-epitope in compare with control group (E. coli and PBS) (Fig. 4B).

\section{DISCUSSION}

Acinetobacter baumannii is difficult to manage due to its fast acquisition of antimicrobial resistance. Recently, WHO has introduced A. baumannii as a highly dangerous pathogen that threat human society. As well due to resistance to numerous antibiotics available considerate as bioterrorism organism (30). Vaccine production against it is a promising alternate and cost-effective strategy to prevent extreme- drugresistant infections. However, no licensed vaccine is available yet in the market and numerous efforts have been made to introduce an actual vaccine against $A$. baumannii. In the past few years, reverse vaccinology is highly regarded for designing vaccines against various diseases, particularly infections $(31,32)$. Using immune-informatics tools for prediction of immunogenic antigens as well as epitope mapping of B- and T-cells with high accuracy can be effective in developing actual and safe vaccine against diseases. Several studies have shown that immunogenic $\mathrm{B}$ - and T-cell multi-epitope are able to provide pro- tective immunity against various bacterial pathogens such as $S$. aureus $(33,34)$ and Brucella abortus $(35)$. Therefore, in this research, we targeted a multi-epitope protein against $A$. baumannii strains. The $A$. baumannii genome encoded over 1,500 proteins that any of which alone or in combination can aid as a potential target for vaccine candidate (36). The Outer membrane protein A $(38 \mathrm{KDa})$ appears to be one of the highly immunogenic protein and an effective candidate for controlling A. baumannii infections $(37,38)$. In our previous study, high antibody titers against OmpA in the serum of recovering ICU patients and staff supports the role of this antigen in A. baumannii infections (27). The outer membrane protein A among different clinical isolates of A. baumannii is very conserved, while the lowest homology with human proteome. Many studies, however, have used OmpA to develop antibody protection in animal models $(39,40)$ but it is not sufficient by itself for protection against $A$. baumannii infection (31). Another very important antigen in Gram-negative bacteria is BAM ( $\beta$-Barrel Assembly Machine) complex. The BAM complex (BamA, BamB, BamC, BamD and BamE) in A. baumannii is highly conserved due to its essential role in outer membrane protein assembly. More recently, Singh at al. have been reported significant immune-protective effects of BamA in a mouse pneumonia model which was created with clinically isolated lethal MDR A. bumannii (41). According to the present study, we designed a novel multi-epitope protein contains selected epitopes

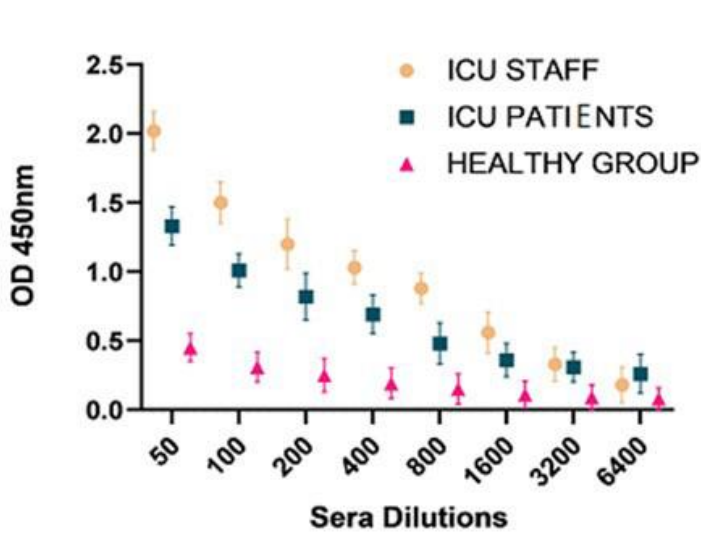

A

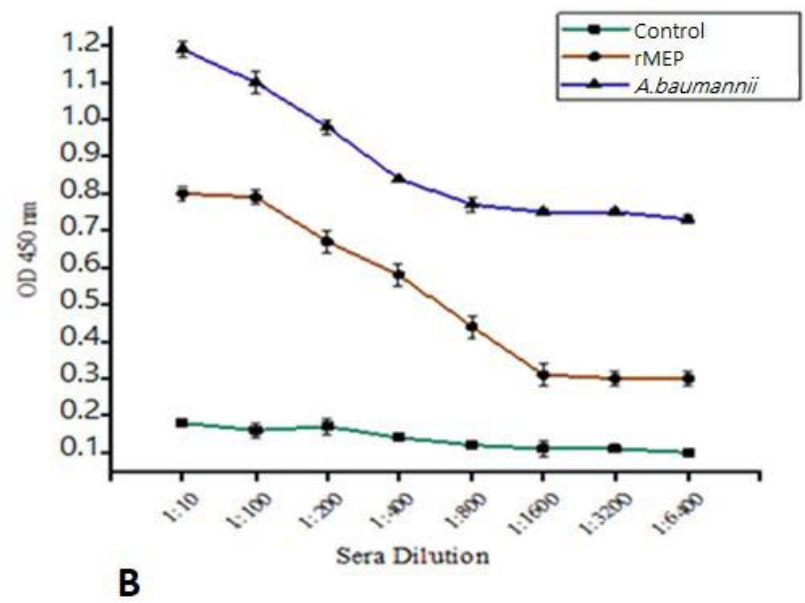

Fig. 4. ELISA analysis. A) The specific total antibody titer in different dilution of ICU staff, patients and healthy group sera in reaction with A. baumannii using ELISA assay. B) Comparative analysis of specific total antibody titer of sera in reaction with A. baumannii, recombinant multi- epitope protein(rMEP) and control group using ELISA assay. Data signifies Mean of the $\mathrm{OD} 450 \pm \mathrm{SD}$ with $\mathrm{P}>0.001$. 
of OmpA, BamA, BamB, BamC, BamD and BamE proteins. According to Ren et al. study designed multi-epitope vaccine with FilF and NucAB antigens could induce high levels of $\mathrm{IgG}$ antibodies and provide potent protection (88.9\%) against lethal doses of $A$. baumannii (22). In further work by Singh et al. a multi-epitope subunit protein of BamA has shown high levels of protection in mice against A. baumanni (14).

To determine the $\mathrm{B}$-cell and $\mathrm{T}$-cell epitopes from selected virulence factors, we used the immune-informatics tools. Since humoral immune is crucial in protection against $A$. baumannii infection, multi-epitope protein was composed of linear B-cell epitopes and CD4+T-cell epitopes to develop an effective antigen. Furthermore, the MHC I and MHC II binding epitopes were designed specifically for the HLA-DRB1*11, 13, 15, 03, 04 alleles because of the high prevalence in the Iranian population (42). Consequently, based on antigenicity, sensitivity, toxicity, and solubility, 14 B- and T-cell epitopes were selected with the best scores. They were linked using a most widely flexible GGGGS linker so that no new epitopes were created. The small size of these amino acids provides flexibility, and allows for mobility of the connecting functional domains (43). The in silico results of this research showed that our multi-epitope protein named rMEP is stable, highly antigenic. To optimize protein expression in E. coli, some parameters, including Codon Adaptation Index (CAI), Codon Frequency Distribution (CFD) and GC content of genes were determined. The results confirm that desired protein structure can be fully expressed in the $E$. coli host. The three-dimensional structure of a protein plays a key role in protein binding and function. Therefore, the 3D structure of the rMEP was modeled using the I-TASSER server. Structural quality and stability of the predicted model were evaluated using Ramachandran map, ProSA Z-score and ERRAT quality factor. The high structural quality of the protein was verified by the obtained results. As mentioned previously, recombinant protein was produced in the form of the inclusion body in the E. coli BL21 (DE3). Protein denaturation was performed using urea $8 \mathrm{M}$, to obtain soluble and active form of protein. In this study, the purified protein was refolding to the active form by stepwise urea removal using Amicon-30KDa filter. Circular Dichroism spectrophotometry analysis was performed to evaluate the effect of purification conditions on re- combinant protein refolding. The results showed that after purification, the recombinant protein retained its structure almost correctly, although the percentage of secondary structures changed slightly. Finally the multi epitope DNA sequence with LC521880 accession number was released in the DDBJ data bank.

On the other hand, antibodies, as primary mediators of the immune system, perform various functions to remove antigens and control infections. Antibodies, opsonizing the pathogens and increase phagocytosis activity of macrophages also activate the complement system, leading to lysis of bacteria. In this research the results of ELISA showed high antibody titers in ICU staff and recovering patient's sera that is supports the role of antibody in A. baumannii infections could interact with selected fragments in rMEP. In previous study we showed that predicted peptides in OmpA protein from A. baumannii could be the efficient epitopes as part of novel vaccine design in animal study (44). Also, to confirm the efficiency of peptides in vaccine design, researchers have been able to use peptides similar to other bacterial components for this purpose. For example, mimicking lipopolysaccharide as a potential vaccine candidate against Vibrio cholerae serogroup O1 (45). Therefore, obtaining effective peptides to stimulate the immune system is one of the most cost-effective and safe methods for vaccine design.

In conclusion, we designed and evaluated a novel protein consisting of high score $\mathrm{B}$ and $\mathrm{T}$ cell epitopes from crucial virulence factors OmpA, Bam A, Bam B, BamC, Bam D, Bam E belong to A. baumannii. Also the sera of the ICU staff and recovering patients with a history of $A$. baumannii infections have been able to interact with this protein.

\section{REFERENCES}

1. Prashanth K, Badrinath S. Nosocomial infections due to Acinetobacter species: clinical findings, risk and prognostic factors. Indian J Med Microbiol 2006; 24 : 39-44.

2. Peleg AY, Seifert H, Paterson DL. Acinetobacter baumannii: emergence of a successful pathogen. Clin Microbiol Rev 2008; 21: 538-582.

3. Shields RK, Clancy CJ, Gillis LM, Kwak EJ, Silveira FP, Massih RC, et al. Epidemiology, clinical characteristics and outcomes of extensively drug-resistant Acinetobacter baumannii infections among solid organ 
transplant recipients. PLoS One 2012; 7(12): e52349.

4. Perez F, Hujer AM, Hujer KM, Decker BK, Rather PN, Bonomo RA. Global challenge of multidrug-resistant Acinetobacter baumannii. Antimicrob Agents Chemother 2007; 51: 3471-3484.

5. Mishra RP, Oviedo-Orta E, Prachi P, Rappuoli R, Bagnoli F. Vaccines and antibiotic resistance. Curr Opin Microbiol 2012; 15: 596-602.

6. Bentancor LV, Routray A, Bozkurt-Guzel C, Camacho-Peiro A, Pier GB, Maira-Litrán T. Evaluation of the trimeric autotransporter Ata as a vaccine candidate against Acinetobacter baumannii infections. Infect Immun 2012; 80: 3381-3388.

7. Golshani M, Rafati S, Jahanian-Najafabadi A, Nejati-Moheimani M, Siadat SD, Shahcheraghi F, et al. In silico design, cloning and high level expression of L7/ L12-TOmp31 fusion protein of Brucella antigens. Res Pharm Sci 2015; 10: 436-445.

8. Singh R, Garg N, Shukla G, Capalash N, Sharma P. Immunoprotective efficacy of Acinetobacter baumannii outer membrane protein, FilF, predicted in silico as a potential vaccine candidate. Front Microbiol 2016; 7: 158.

9. Russo TA, Beanan JM, Olson R, MacDonald U, Cox AD, St Michael F, et al. The K1 capsular polysaccharide from Acinetobacter baumannii is a potential therapeutic target via passive immunization. Infect Immun 2013; 81: 915-922.

10. Noori E, Rasooli I, Owlia P, Mousavi Gargari SL, Ebrahimizadeh W. A conserved region from biofilm associated protein as a biomarker for detection of Acinetobacter baumannii. Microbia Pathog 2014; 77 : 84-88.

11. McConnell MJ, Rumbo C, Bou G, Pachón J. Outer membrane vesicles as an acellular vaccine against Acinetobacter baumannii. Vaccine 2011; 29: 57055710.

12. Nie D, Hu Y, Chen Z, Li M, Hou Z, Luo X, et al. Outer membrane protein $\mathrm{A}(\mathrm{OmpA})$ as a potential therapeutic target for Acinetobacter baumannii infection. J Biomed Sci 2020; 27: 26

13. Rasooli I, Abdolhamidi R, Jahangiri A, Darvish Alipour Astaneh S. Outer membrane protein, Oma87 prevents Acinetobacter baumannii infection. Int J Pept Res Ther 2020; 1-8.

14. Huang W, Yao Y, Wang S, Xia Y, Yang X, Long Q, et al. Immunization with a $22-\mathrm{kDa}$ outer membrane protein elicits protective immunity to multidrug-resistant Acinetobacter baumannii. Sci Rep 2016; 6: 20724

15. Gaddy JA, Tomaras AP, Actis LA. The Acinetobacter baumannii 19606 OmpA protein plays a role in biofilm formation on abiotic surfaces and in the interaction of this pathogen with eukaryotic cells. Infect Immun 2009; 77: 3150-3160.
16. Moon DC, Choi CH, Lee JH, Choi CW, Kim HY, Park JS, et al. Acinetobacter baumannii outer membrane protein A modulates the biogenesis of outer membrane vesicles. J Microbiol 2012; 50: 155-160.

17. Choi CH, Lee EY, Lee YC, Park TI, Kim HJ, Hyun $\mathrm{SH}$, et al. Outer membrane protein 38 of Acinetobacter baumannii localizes to the mitochondria and induces apoptosis of epithelial cells. Cell Microbiol 2005; 7 : 1127-1138.

18. Hagan CL, Wzorek JS, Kahne D. Inhibition of the $\beta$-barrel assembly machine by a peptide that binds BamD. Proc Natl Acad Sci U S A 2015; 112: 2011-2016.

19. Hagan CL, Silhavy TJ, Kahne D. $\beta$-Barrel membrane protein assembly by the Bam complex. Annu Rev Biochem 2011; 80: 189-210.

20. Knowles TJ, Scott-Tucker A, Overduin M, Henderson IR. Membrane protein architects: the role of the BAM complex in outer membrane protein assembly. Nat Rev Microbiol 2009; 7: 206-214.

21. Guo SJ, Ren S, Xie YE. Evaluation of the protective efficacy of a fused OmpK/Omp22 protein vaccine candidate against Acinetobacter baumannii infection in mice. Biomed Environ Sci 2018; 31: 155-158

22. Ren S, Guan L, Dong Y, Wang C, Feng L, Xie Y. Design and evaluation of a multi-epitope assembly peptide vaccine against Acinetobacter baumannii infection in mice. Swiss Med Wkly 2019; 149: w20052.

23. Badmasti F, Ajdary S, Bouzari S, Fooladi AA, Shahcheraghi F, Siadat SD. Immunological evaluation of OMV (PagL)+ Bap (1-487aa) and AbOmpA (8-346aa)+ Bap (1-487aa) as vaccine candidates against Acinetobacter baumannii sepsis infection. Mol Immunol 2015; 67(2 Pt B): 552-558

24. Bambini S, Rappuoli R. The use of genomics in microbial vaccine development. Drug Discov Today 2009; 14: $252-260$

25. Soria-Guerra RE, Nieto-Gomez R, Govea-Alonso DO, Rosales-Mendoza S. An overview of bioinformatics tools for epitope prediction: implications on vaccine development. J Biomed Inform 2015; 53: 405-414.

26. Mohammadi F, Goudarzi H, Hashemi A, Yousefi Nojookambari N, Khoshnood S, Sabzehali F. Detection of ISAbal in Acinetobacter baumannii strains carrying OXA genes isolated from Iranian burns patients. Arch Pediatr Infect Dis 2016; 5(2); e39307.

27. Nafarieh T, Bandehpour M, Hashemi A, Taheri S, Yardel V, Jamaati $\mathrm{H}$, et al. Identification of antigens from nosocomial Acinetobacter baumannii clinical isolates in sera from ICU staff and infected patients using the antigenome technique. World J Microbiol Biotechnol 2017; 33: 189.

28. Jositsch G, Papadakis T, Haberberger RV, Wolff M, Wess J, Kummer W. Suitability of muscarinic acetylcholine receptor antibodies for immunohistochemistry 
evaluated on tissue sections of receptor gene-deficient mice. Naunyn Schmiedebergs Arch Pharmacol 2009; 379: 389-395.

29 Ho BK, Brasseur R. The Ramachandran plots of glycine and pre-proline. BMC Struct Biol 2005; 5: 14.

30. Xie R, Zhang XD, Zhao Q, Peng B, Zheng J. Analysis of global prevalence of antibiotic resistance in Acinetobacter baumannii infections disclosed a faster increase in OECD countries. Emerg Microbes Infect 2018; 7: 31.

31. Chiang MH, Sung WC, Lien SP, Chen YZ, Lo AF, Huang $\mathrm{JH}$, et al. Identification of novel vaccine candidates against Acinetobacter baumannii using reverse vaccinology. Hum vaccin Immunother 2015; 11: 1065 1073.

32. Gomez G, Pei J, Mwangi W, Adams LG, Rice-Ficht A, Ficht TA. Immunogenic and invasive properties of Brucella melitensis $16 \mathrm{M}$ outer membrane protein vaccine candidates identified via a reverse vaccinology approach. PLoS One 2013; 8(3): e59751.

33. Ahmadi K, Pouladfar G, Kalani M, Faezi S, Pourmand MR, Hasanzadeh S, et al. Epitope-based immunoinformatics study of a novel Hla-MntC-SACOL0723 fusion protein from Staphylococcus aureus: induction of multi-pattern immune responses. Mol Immunol 2019; 114: 88-99.

34. Esmailnia E, Amani J, Gargari SLM. Identification of novel vaccine candidate against Salmonella enterica serovar Typhi by reverse vaccinology method and evaluation of its immunization. Genomics 2020; 112: 33743381.

35. Sadeghi Z, Fasihi-Ramandi M, Bouzari S. Evaluation of immunogenicity of novel multi-epitope subunit vaccines in combination with poly I:C against Brucella melitensis and Brucella abortus infection. Int Immunopharmacol 2019; 75: 105829.

36. Chopra S, Ramkissoon K, Anderson DC. A systematic quantitative proteomic examination of multidrug resistance in Acinetobacter baumannii. J Proteomics 2013; 84: $17-39$.

37. Lin L, Tan B, Pantapalangkoor P, Ho T, Hujer AM,
Taracila MA, et al. Acinetobacter baumannii rOmpA vaccine dose alters immune polarization and immunodominant epitopes. Vaccine 2013; 31: 313-318.

38. Fajardo Bonin R, Chapeaurouge A, Perales J, da Silva JG Jr, do Nascimento HJ, D'Alincourt Carvalho, et al. Identification of immunogenic proteins of the bacterium Acinetobacter baumannii using a proteomic approach. Proteomics Clin Appl 2014; 8: 916-923.

39. Ansari H, Doosti A, Kargar M, Bijanzadeh M, Jaafarinia M. Cloning of ompA gene from Acinetobacter baumannii into the eukaryotic expression vector pBudCE4.1 as DNA vaccine. Indian J Microbiol 2018; 58: $174-181$.

40. Bolourchi N, Shahcheraghi F, Shirazi AS, Janani A, Bahrami F, Badmasti F. Immunogenic reactivity of recombinant PKF and AbOmpA proteins as serum resistance factors against sepsis of Acinetobacter baumannii. Microb Pathog 2019; 131: 9-14.

41. Singh R, Capalash N, Sharma P. Immunoprotective potential of BamA, the outer membrane protein assembly factor, against MDR Acinetobacter baumannii. Sci Rep 2017; 7: 12411.

42. Yari F, Sobhani M, Sabaghi F, Zaman-Vaziri M, Bagheri N, Talebian A. Frequencies of HLA-DRB1 in Iranian normal population and in patients with acute lymphoblastic leukemia. Arch Med Res 2008; 39: 205 208.

43. Chen X, Zaro JL, Shen WC. Fusion protein linkers: property, design and functionality. Adv Drug Deliv Rev 2013; 65: 1357-1369.

44. Mehdinejadiani K, Bandehpour M, Hashemi A, Ranjbar MM, Taheri S, Jalali SA, et al. In silico design and evaluation of Acinetobacter baumannii outer membrane protein $\mathrm{A}(\mathrm{OmpA})$ antigenic peptides as vaccine candidate in immunized mice. Iran J Allergy Asthma Immunol 2019; 18: 655-663.

45. Ghazi FMP, Gargari SLM. Synthetic peptides mimicking lipopolysaccharide as a potential vaccine candidate against Vibrio cholerae serogroup O1. Iran J Microbiol 2017; 9: 244-250. 